\title{
Lifetime Maximization in Wireless Sensor Networks Based on Using Data Compression
}

\author{
Safa Khudair Leabi \\ Dept. of Information Technology, Middle Technical \\ University, Technical College of Management \\ Baghdad, Iraq
}

\author{
Turki Younis Abdalla \\ Dept. of Computer Engineering, College of \\ Engineering, University of Basrah \\ Basrah, Iraq
}

\begin{abstract}
In recent years, the demand and development of multimedia product expands increasingly fast, sharing out networks bandwidth and appliances memory. Therefore, data compression theory becomes the major considerable for reducing datum volume so as to economize extra transmission bandwidth and hardware sharing. Energy limitations have become fundamental challenge for designing wireless sensor networks. Network lifetime represent the most important and interested metric. This paper proposes data compression methods for maximizing lifetime of the network. Two lossless compression methods are proposed for compressing patients ECG data. The first is Huffman coding and the second is arithmetic coding. Dijkstra routing has been used as the main routing protocol. A comparison has been made for the two compression methods. Simulations demonstrate that Huffman coding has superior performance against arithmetic coding. It shows an increase in network lifetime of about $63 \%$ while arithmetic coding shows lifetime increase of about $16.76 \%$. Results show the effectiveness of the Huffman coding method for maximizing WSNs lifetime.
\end{abstract}

\section{Keywords}

Wireless sensor networks, network lifetime, data compression, ECG data, Huffman coding, arithmetic coding.

\section{INTRODUCTION}

Recent years advances show serious progress in wireless networking. The progress and growth in wireless communication technology have made WSNs attractive for multiple application areas, such as medical and health, security surveillance, habitat monitoring, military reconnaissance, disaster management, industrial automation, etc. [1-4]. The development of small and ubiquitous WSNs computing devices is ultimately required. WSNs are comprised of considerable number of limited capabilities sensor nodes with one or more high capability base stations. Each sensor node is a small embedded system, low-power, low-cost, multi-functional [3]. Each sensor node performs several functions: sensing, data processing, and communication. Sensor nodes perform wireless communications with each other in order for delivering gathered data to base station. The development of ubiquitous, inexpensive, small and low-power computing devices became available through miniaturization technologies [3]. Due to this, using multi-hop communication help to reduce transmission distance as well as increasing network lifetime. Every node consists of four parts: a processer, sensor, transceiver, and battery. Nodes involve bounded power source with abilities of sensing, datum processing along with communication. The onboard sensors collect datum about the environment through event driven or continuous working mode. The gathered datum may be temperature, pressure, acoustic, pictures, videos, etc. The gathered datum is then transferred across the network in order to form a global monitoring view for objects $[5,6]$.

Since bounded energy source is involved, energy exhaustion is the most important metric for WSNs. In order for maximizing networks lifetime, energy exhaustion must be well managed [7, 8].

Since the main source of power exhaustion of wireless nodes spent for communication between each other, the network designers solves the energy consumption issues by reducing the amount of transmitted data using data aggregation techniques or data compression techniques [9-12].Data Compression has considered as important for the WSNs applications because the existence of high datum for broadcasting across WSN. Data compression methods involve reducing the size of gathered information before transmission. Decompression of data is the responsibility of the base station. In compression techniques, it is important that there is no information lost and individual readings are retained.

In this paper, Huffman coding for compressing patients ECG data is proposed. The main goal of the proposed compression technique is to reduce energy exhaustion and prolonging wireless sensor network lifetime.

This paper is organized as follows: related work is presented in section 2. Section 3 describe the proposed routing technique. Simulation settings are presented in section 4. Simulation results and discussion are presented in section 5 . Conclusion is presented in section 6 .

\section{RELATED WORK}

One of the primary considerations in wireless sensor networks is the design of energy efficient system. Data compression methods reduce files size for transmission. Data compression can solve power exhaustion issue in these networks and maximize the lifetime of the overall network. Prolonging network lifetime has gained significant interest in latest years.

Byoungyup et al. [13] proposed a modified Huffman coding called sHuffman for WSNs. The efficiency of the suggested method has evaluated and compared with different compression methods. Temperature data sets are used for simulation. Simulation results showed decrease in network traffic of about $40 \%$ and increase in the network lifetime of about $30 \%$.

The work proposed by Henry et al. [14] presented a lightweight data compression scheme depending on 
conventional Huffman coding method for WSNs. Proposed method efficiency has evaluated and compared with LEC and ALEC. The proposed scheme provide compression ration between $46 \%$ and $82 \%$ for different temperature and humidity data sets.

Rachelin and Selvaraju [15] proposed a simple modified Huffman coding scheme for WSNs. The proposed scheme combine the static Huffman and adaptive Huffman coding. The proposed algorithm has better compression ratio and less computation time than adaptive Huffman coding. AODV is used as the based routing protocol. Result is compared with data without compression. Results showed a decrease in energy consumption and increase in network lifetime.

The work proposed by Asral B. and Nor A. [16] presents a performance comparison of data compression in WSNs. They consider Huffman coding and LZW coding in this work. Experimental results showed that Huffman coding has better performance than LZW coding in term of compression ratio and computation time. Huffman coding provides compression ratio of $43 \%$ and less computation time.

Maher et al. [17] proposed a MAS lossless data compression technique for the use in wireless sensor networks. Different data set is used for simulation. Simulation is carried out in OMNET++ simulation platform. The proposed MAS compression method is compared with S-LZW and K-RLE methods. Simulation results showed that MAS compression method has risen energy saving of about $54 \%$ for all test data sets. Also, simulation results showed that MAS compression method is better than the other two methods in terms of compression ratio, compression speed, memory requirements and energy savings.

The work proposed by Dragoș et al. [18] presented a data compression scheme based on Huffman coding for wireless sensor networks. The proposed algorithm consists of prediction algorithm, static Huffman algorithm, data aggregation technique, and a method of transition from bitwise to byte wise approach, BAT. The work is tested in visual basic platform and practical microcontroller platform. Results showed a decrease in node energy consumption and number of bytes transmitted.

Jonathan et al. [19] proposed an adaptive lossless data compression for wireless sensor networks. The proposed data compression method uses multiple code options. The proposed method uses Huffman coding as the base coding method. The proposed compression schemes allow compression to dynamically adjust to a changing source using two Huffman coding methods. The proposed method is compared with LEC and S-LZW methods. It showed a compression performance up to $74 \%$ using real world data set.

The work proposed by Phayong et al. [20] presents a simple data compression for WSNs. The proposed algorithm is a modified Huffman algorithm. The proposed algorithm is evaluated on practical testbed. Experimental results demonstrate that the suggested algorithm could alleviate both memory and energy constraints. It provides a compression ratio of about $50.25 \%$ for temperature and humidity data set. Also they apply a recompression in the master node which provides a compression ratio of about $4.94 \%$. They suggest using this algorithm in real world WSNs.

Andreas et al. [21] proposed a lightweight adaptive Huffman coding for wireless sensor networks. The proposed adaptive Huffman coding operates on trees code of Huffman with a limited number of nodes. For energy evaluation, simulation is carried out in MSPsim and COOJA. Simulation results showed that the proposed method save about of $50 \%$ of radio energy for some data sets. It also showed the proposed method reduce energy consumption even when small size data set is used.

The work proposed by Eugene et al. [22] presents a lossy KRLE compression method for wireless sensor networks. The proposed algorithm is a modified run length encoding with the $\mathrm{K}$ value equal to 2 . The proposed algorithm is tested on MSP430 microcontroller. The proposed algorithm is compared with the RLE and S-LZW algorithms. The proposed algorithm provides a better compression ratio of about $56 \%$ for temperature data set in comparison with the other two methods. Energy consumption evaluation of the previous data compression algorithms is carried out using WSim, which is a cycle accurate hardware platform simulator. Simulation results showed that energy consumption is increased with the increase of the compression ratio.

\section{THE PROPOSED METHOD}

The information of Electrocardiogram (ECG) contributes in the diagnosing process of cardiac disease for patients. The ECG is a graphical report depicted by electrical signal that reflect contractile effectiveness of the heart [23]. To get sufficient inspection for patients, the size of the ECG data must be large. ECG report give detailed facts about the heart rate, rhythm, and morphology.

Owing to the fact that nodes involve limited capabilities and large size of ECG datum, compression techniques is very necessary and important for ECG application in WSNs. The main objective of ECG compression is to involve reduced data transfer so as for minimizing energy exhaustion along with maximizing total network lifetime. In this section, we proposed lossless static Huffman coding technique for ECG compression in WSNs. The choice of this technique is due to its simplicity, high compression ratio, and reduced computation time. This technique is compared with other techniques like arithmetic coding. An appropriate compression for ECG data is performed in each node and transfers as binary data. The decompression is performed in the sink to gather the source information.

\subsection{Huffman Coding}

Huffman coding was first proposed by David Huffman in 1952 [24]. Huffman method involves specifying binary codes to symbols. Total number of bits required for encoding symbols is minimized depending on probability or frequency of appearance (Weight) of source symbols. Huffman coding method tabulates symbols and their weights, and then generates adequate coding for these symbols. Big weights symbols get fewer bits for their coding.

Huffman encoding firstly tabulates whole symbols towards descending order depending on their probabilities. Next stage is fabricating a tree for symbols from the bottom up by using a procedure of sequential steps. For every step select symbols that handle lowest probability values, add them into tree, delete them from the weighted list, and replace them with helpful symbols representing them. The tree is complete when all symbols in the list are covered. To get encoding Huffman code, traverse the constructed binary tree [25]. The Huffman algorithm can be described as follows. Huffman technique of coding involves fabricating a tree of nodes first. The Huffman tree can be constructed as follows. 
1. Every character generate node along with adding it into priority queue.

2. While still multiple nodes in queue.

a. Put aside lowest prospect node double for getting couple nodes.

b. Generate fresh node from offspring couple nodes along with prospect equal to sum of couple nodes' prospects.

c. Next step is adding fresh node into queue.

3. The lasting node represent root. Hence, tree has complete.

To constitute Huffman encodings for these symbols and weights, go over the fabricated binary tree from the bottom up along with addressing zero for one branch and one for the other branch with every node. Accumulating binary numbers construct final codes.

\subsection{Arithmetic Coding}

Arithmetic coding is a mechanism for encoding and decoding information. Arithmetic coding allows the messages information in a message sequence to be combined so that it shares the same bits. Arithmetic coding encodes blocks of symbols instead of encoding each symbol. The major drawback of the arithmetic coding is it involves high computation time because of many multiplications and divisions are needed.

Arithmetic coding idea is similar to block Huffman coding idea and it specifies a codeword to whole blocks of symbols and not to individual symbols [25-28]. For compressing a file, firstly it get characters from this file one after one. Then specify intervals depending on probability. Intervals can be arranged vertically with two limits or horizontally with one limit. High probability character narrow the interval in wide rather than other characters. Slimmer interval involves more bits and continuously increasing depending on the method. Hence, they get less bits against other characters.

In comparison to computationally simple Huffman coding, both arithmetic coding method are comes with significantly higher complexity. The main reason of not using of complex arithmetic coding in systems of data compression is the patents restrictions.

Arithmetic coding involves the following procedure.

1) Stand for every string (stg) of length $\mathrm{m}$ with individual interval [LF, RT) among [0,1).

2) (stg) occurrence' prospect stand for width rt-1 upon interval [LF,RT).

3) Represent interval [LF, RT) using any number. Named tag inside mid open interval. Tag might selected as $(\mathrm{LF}+\mathrm{RT}) / 2$.

4) Tag significant bits (.g1g2g3) represent code of (stg).

\section{SIMULATION SETUP AND CONFIGURATIONS}

Simulation is carried out in MATLAB. For the proposed ECG compression based routing, two scenarios for two topological areas A1 and A2 are considered. A 100 nodes are randomly scattered for every topological areas. The topological areas $\mathrm{A} 1$ and $\mathrm{A} 2$ have the dimension of $100 \mathrm{~m} \times 100 \mathrm{~m}$ for area A1 and $200 \mathrm{~m} \times 50 \mathrm{~m}$ for area A2. One base station "Sink" has been used for each topological area. The position of the sink is (90m, 90m) for topological area A1, and is $(180 \mathrm{~m}, 45 \mathrm{~m})$ for topological area A2. Every node operate with maximum transmission range equal to $30 \mathrm{~m}$. Each node has initial energy equal to 250J. The packet length is computed for compressed files. Ten ECG data files are considered for simulation. ECG files used are with 500x500 data. For ECG data used, minimum file size is $12 \mathrm{~KB}$ and maximum file size is $13 \mathrm{~KB}$. A sample of ECG data is depicted in figure 1. The proposed routing technique utilized with first order radio model proposed by Heinzelman et al. [29], which is largely used in the field of evaluating routing techniques for WSNs. This model is shown in the following equations.

$$
E_{T X}\left(p k t_{\text {lengt } h}\right)=E_{\text {elec }} * p k t_{\text {lengt } h}+E_{\text {amp }} * p k t_{\text {lengt } h} * d^{2}
$$

$E_{R X}\left(p k t_{\text {lengt } h}\right)=E_{\text {elec }} * p k t_{\text {lengt } h}$

Where, $E_{T x}$ and $E_{R x}$ are the energy consumption for transmitting and receiving respectively. pkt $_{\text {length }}$ is the number of bits per packet. $\mathrm{d}$ is the distance from sender node to receiver node. $\mathrm{E}_{\text {elec }}$ is the per bit energy dissipation for transmitting or receiving for electrical circuitry. $E_{a m p}$ is the per bit per meter square energy dissipation. The values of $E_{\text {elec }}$ and $E_{a m p}$ used for simulation are $50 \mathrm{~nJ} / \mathrm{bit}$ and $100 \mathrm{pJ} / \mathrm{bit} / \mathrm{m} 2$, respectively.

Dijkstra routing has been used as the main routing protocol. The routing protocol randomly select an ECG data file from the ten ECG data files stored previously. The routing protocol compress the selected ECG file and send it through routing path. The other nodes in the routing path just forward the compressed packet toward the sink. The sink decompress the compressed ECG file to reconstruct the ECG data file. Two compression methods are considered which are Huffman Coding and Arithmetic Coding.

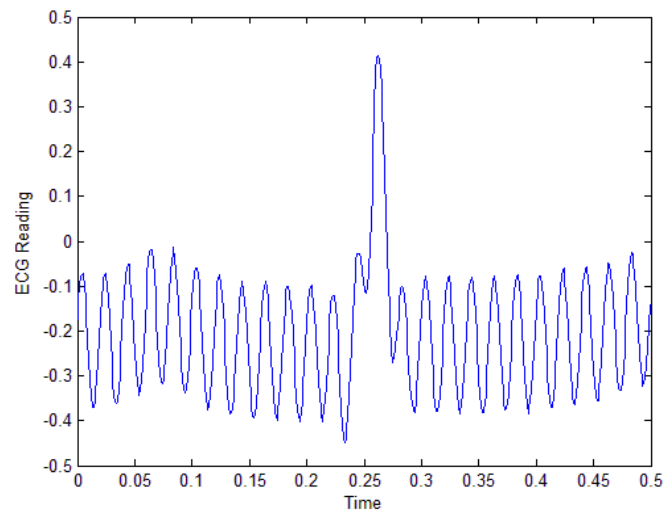

Fig 1. A sample of ECG Data

\section{SIMULATION RESULTS AND DISCUSSION}

Simulation is carried out for the two scenarios. Huffman coding and arithmetic coding based Dijkstra routing are considered for simulation. Number of alive nodes in each round has used to give the indication about the lifetime of the WSNs. Minimum remaining energy is used to give indication about energy consumption. A comparison has been made for the two coding methods by means of overall network lifetime. Network lifetime refer to period from starting work of network till the first sensor node die or exhaust its energy.

Figure 2(a, b) depicts simulation results for the two topological areas, for number of still alive nodes in each round till network partitioning. From this figure, it can be seen that the Huffman coding is better than the arithmetic coding. It shows an increase in network lifetime of about $63.23 \%$ for 
area $\mathrm{A} 1$ and $63.19 \%$ for area $\mathrm{A} 2$. While arithmetic coding shows lifetime increase of about $16.76 \%$ for area A1 and $16.69 \%$ for area A2. Results show the improvement of the Huffman coding in comparison with the arithmetic coding based routing. In addition, this figure reflects the effectiveness of data compression on the lifetime of WSNs. Network partition feature has been activated for the simulation. Network partitioning is work out when any of the 100 deployed sensor nodes has not find a neighbor nodes to send data packet. This is due to the dyed sensor nodes. Hence, simulation is stopped when network partition is occurred. Table 1 details the overall network lifetime and network partitioning time for the two methods and for the two topological areas A1 and A2.

Figure 3(a, b) illustrates the network minimum remaining energy till network partition in each round and for the two topological areas $\mathrm{A} 1$ and $\mathrm{A} 2$, respectively, and also a comparison between Huffman coding and arithmetic coding. It is obvious from these figures, that the minimum remaining energy of the proposed Huffman coding is higher than the arithmetic coding. These results reflect energy exhaustion has reduced. The proposed Huffman coding results in reduced energy exhaustion along with maximized total network lifetime.

Figure 4(a, b) with figure 5(a, b) illustrate consumed energy with reference to nodes that hold id 71 and id 95 for area A1 and for nodes ids 14 and 70 for area A2, respectively. From these figures, consumed energy for Huffman coding is less than the arithmetic coding. The key effect of compression ratio of Huffman coding is higher than the arithmetic coding. This reflect the effectiveness of image compression for reducing energy consumption and prolonging network lifetime.

Figure $6(\mathrm{a}, \mathrm{b})$ with figure $7(\mathrm{a}, \mathrm{b})$ depict simulation time with reference to nodes that hold id 65and id 98 for area $\mathrm{A} 1$ and for nodes ids 55and 95 for area A2, respectively. From these figures, simulation time for Huffman coding is higher than of arithmetic coding. This is due to compression process related to Huffman coding. Nevertheless, in other hand compression ratio of Huffman coding is so higher than arithmetic coding so that ECG files size have reduced significantly. This yields to reduced energy exhaustion along with maximized network lifetime. Table 2 give a detail about the ten files and the compression ratio for both arithmetic coding and Huffman coding.

Table 1: Network Lifetime and Partitioning Time

\begin{tabular}{|c|c|c|c|}
\hline 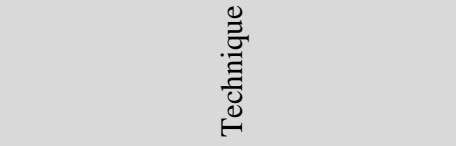 & 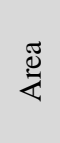 & 节 & : \\
\hline Dijkstra without data compression & A1 & 1003 & 2457 \\
\hline Dijkstra with Arithmetic Coding & A1 & 1205 & 2951 \\
\hline Dijkstra with Huffman Coding & A1 & 2728 & 6682 \\
\hline Dijkstra without data compression & $\mathrm{A} 2$ & 604 & 1500 \\
\hline Dijkstra with Arithmetic Coding & $\mathrm{A} 2$ & 725 & 1802 \\
\hline Dijkstra with Huffman Coding & A2 & 1641 & 4080 \\
\hline
\end{tabular}

Table 2: Files Size and Compression Ratio

\begin{tabular}{|c|c|c|c|c|c|}
\hline 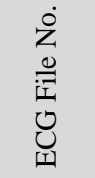 & 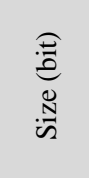 & 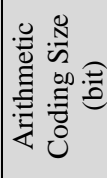 & 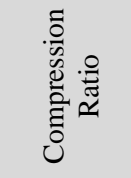 & 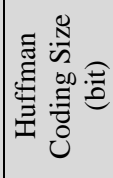 & 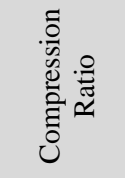 \\
\hline d1.dat & 99800 & 79352 & $20.488 \%$ & 35112 & $64.817 \%$ \\
\hline d2.dat & 99880 & 81352 & $18.550 \%$ & 35096 & $64.861 \%$ \\
\hline d3.dat & 99912 & 82880 & $17.047 \%$ & 35952 & $64.016 \%$ \\
\hline d4.dat & 99800 & 84056 & $15.775 \%$ & 36616 & $63.310 \%$ \\
\hline d5.dat & 99800 & 83336 & $16.496 \%$ & 37368 & $62.557 \%$ \\
\hline d6.dat & 99752 & 83800 & $15.991 \%$ & 37472 & $62.434 \%$ \\
\hline d7.dat & 99760 & 83448 & $16.351 \%$ & 36704 & $63.207 \%$ \\
\hline d8.dat & 99768 & 84576 & $15.227 \%$ & 37240 & $62.673 \%$ \\
\hline d9.dat & 99792 & 83720 & $16.105 \%$ & 37944 & $61.976 \%$ \\
\hline d10.dat & 99960 & 84304 & $15.662 \%$ & 37384 & $62.601 \%$ \\
\hline
\end{tabular}

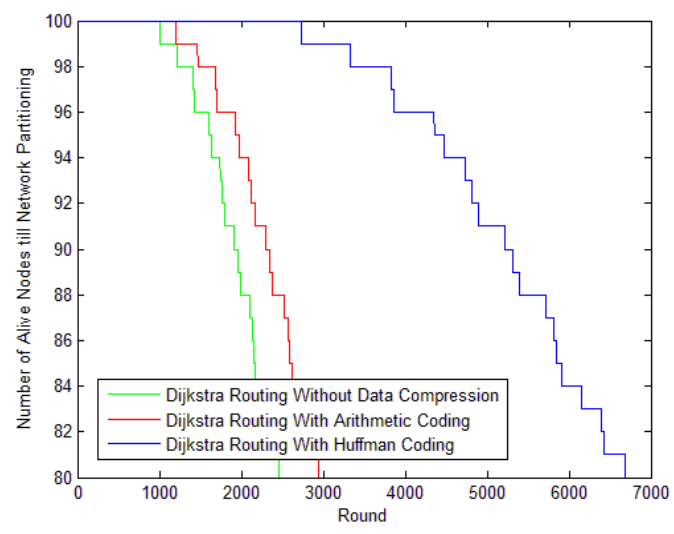

Fig 2(a). Number of alive nodes for area A1

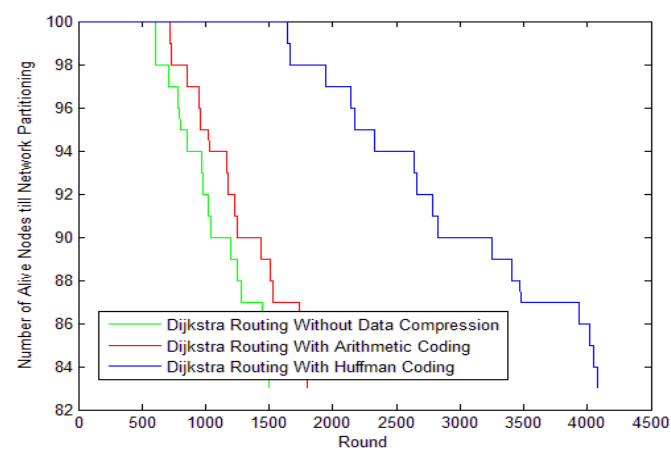

Fig 2(b). Number of alive nodes for area A2

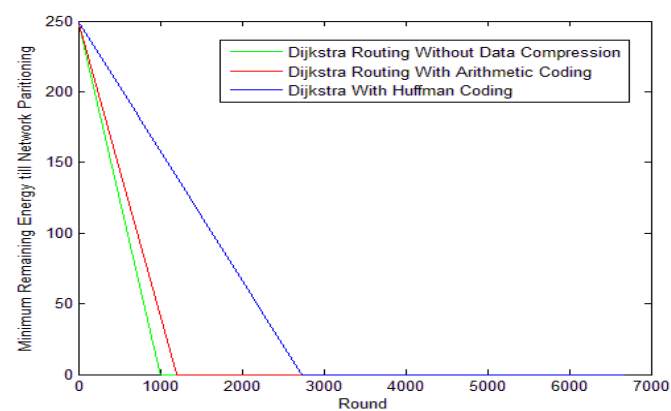

Fig 3(a). Minimum remaining energy for area A1 


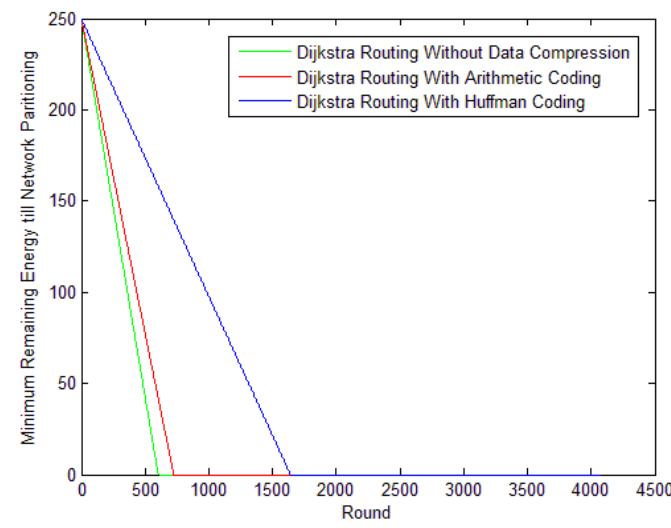

Fig 3(b). Minimum remaining energy for area A2

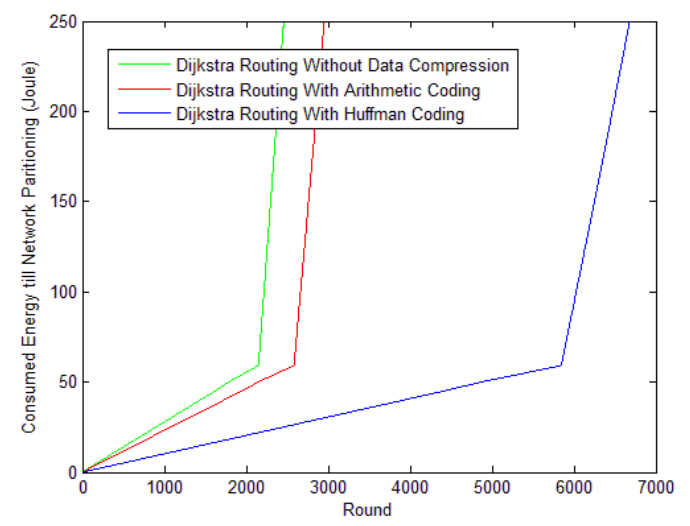

Fig 4(a). Consumed energy for node id (71) for area A1

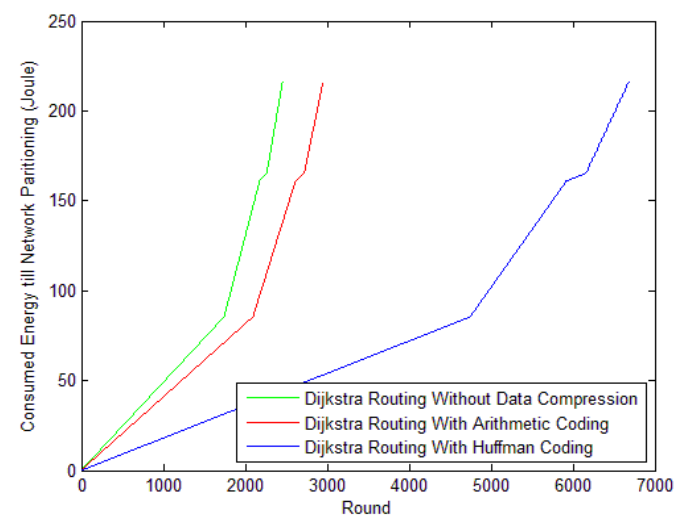

Fig 4(b). Consumed energy for node id (95) for area A1

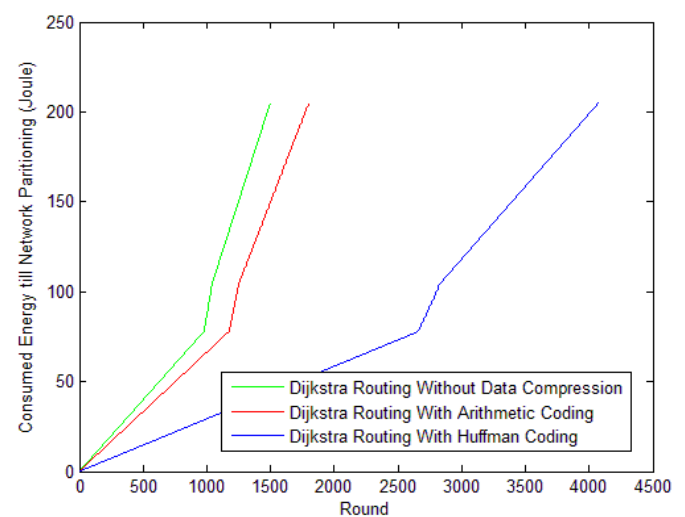

Fig 5(a). Consumed energy for node id (14) for area A2

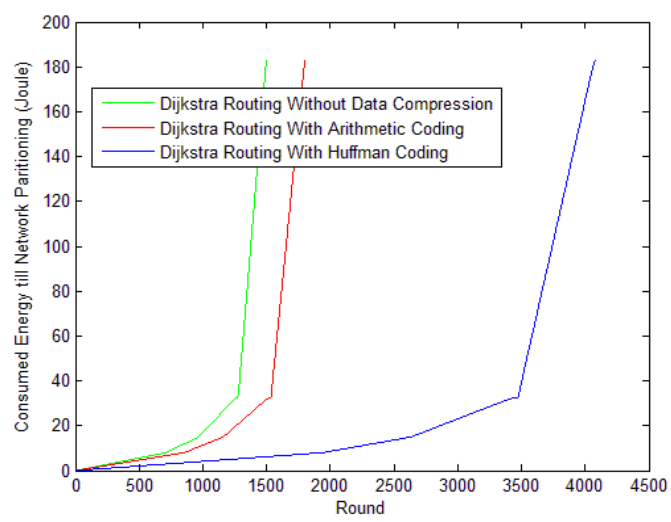

Fig 5(b). Consumed energy for node id (70) for area A2

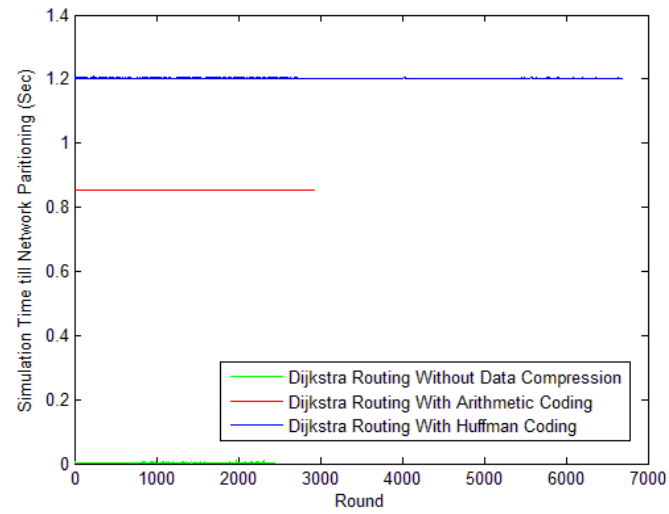

Fig 6(a). Simulation time for node id (65) for area A1

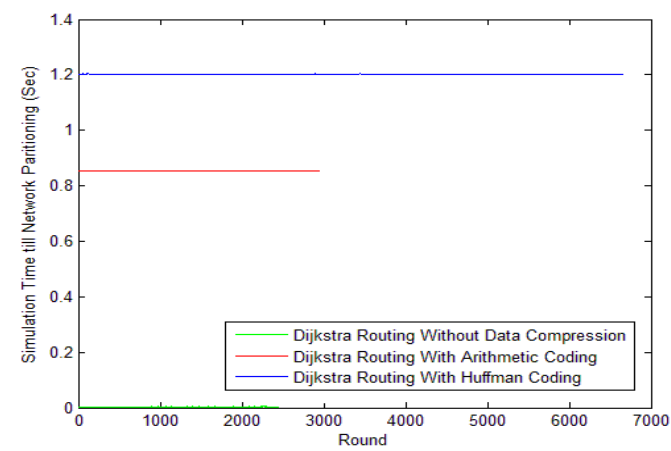

Fig 6(b). Simulation time for node id (98) for area A1

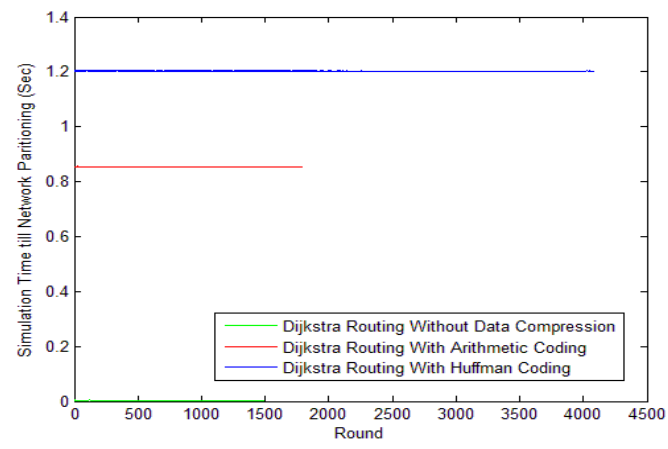

Fig 7(a). Simulation time for node id (55) for area A2 


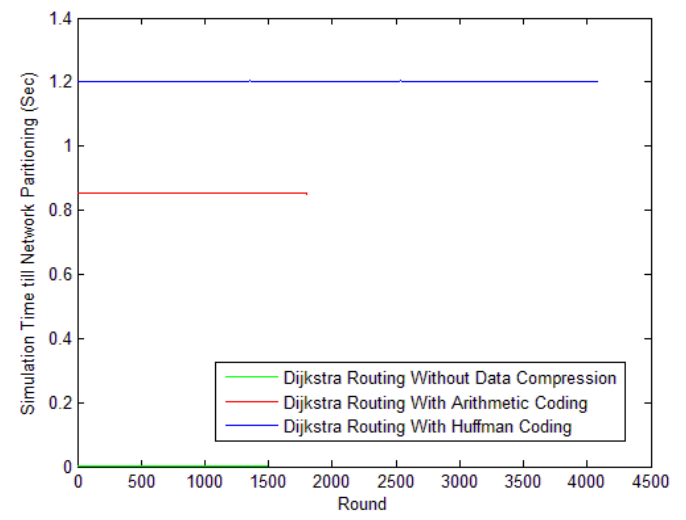

Fig 7(b). Simulation time for node id (95) for area $\mathrm{A2}$

\section{CONCLUSION}

Wireless sensor networks are available with limited source power through their life cycle. Since the battery of the sensor node cannot be replaced or recharged, energy conservation is a crucial issue in the design of the wireless sensor network infrastructure. In this paper, we proposed data compression methods for maximizing the lifetime of the network. Two compression methods are proposed. The first is Huffman coding, and the second is arithmetic coding. The two methods have been used for compressing patients ECG data. Dijkstra routing are used as the main routing protocol. Huffman coding method is used to compress ECG data gathered from sensors due to its simplicity and high compression ratio. Huffman coding is compared with arithmetic coding compression method. Two topological areas are considered which are A1 and A2. Simulation results demonstrate that Huffman coding has superior performance against arithmetic coding. It shows an increase in network lifetime of about $63.23 \%$ for area A1 and $63.19 \%$ for area A2. Arithmetic coding shows lifetime increase of about $16.76 \%$ for area A1 and $16.69 \%$ for area A2. Result figures show the improvement of the Huffman coding in comparison with the arithmetic coding based routing. They reflect the effectiveness of data compression based routing on the lifetime of WSNs.

\section{REFERENCES}

[1] Ian F. Akyildiz and Mehmet Can Vuran, "Wireless Sensor Network", John Wiley \& Sons Ltd., 2010.

[2] A. Hac, "Wireless Sensor Network Designs", John Wiley \& Sons Ltd, 2003.

[3] I. Akyildiz, W. Su, Y. Sankarasubramaniam, and E. Cayirci, "A Survey on Sensor Networks," IEEE Communications Mag., Vol. 40, No. 8, Aug. 2002, pp: 102-114.

[4] Al-Karaki, J.N. Kamal and A.E., "Routing Techniques In Wireless Sensor Networks: A Survey", IEEE Wireless Communication, Vol. 11, 2004, pp:6-28.

[5] Ananthram Swami, Qing Zhao, Yao-Win Hong and Lang Tong, "Wireless Sensor Networks: Signal Processing and Communications Perspectives", John Wiley \& Sons Ltd, 2007.

[6] Amiya Nayak and Ivan Stojmenovic, "Wireless Sensor and Actuator Networks: Algorithms and Protocols for Scalable Coordination and Data Communication", John Wiley \& Sons, Inc., 2010.
[7] C. Hua and T. P. Yum, "Optimal Routing And Data Aggregation For Maximizing Lifetime Of Wireless Sensor Networks", IEEE ACM Transection on Network., Vol. 16, No. 4, pp. 892-903, Aug. 2008.

[8] H. R. Karkvandi, E. Pecht, and O. Yadid, "Effective Lifetime-Aware Routing In Wireless Sensor Networks", IEEE Sensors Journal, Vol. 11, No. 12, pp. 3359-3367, Dec. 2011.

[9] Shailaja Y. Kanawade, Dinesh S. Bhadane, MonaliR.Tarle, and RutushaS.Patel, "A Survey of Data Compression Techniques in Sensor Network", International Journal of Emerging Technology and Advanced Engineering, Vol. 4, Issue 10, pp.415-417, 2014.

[10] ShabanaMehfuz and Usha Tiwari, "Recent Strategies of Data compression in Wireless Sensor Networks", Proceedings of International Conference on Advances in Electrical \& Electronics, pp.653-658, 2013.

[11] S. Mohamed Saleem and P. Sasi Kumar, "Evaluating Effectiveness of Data Transmission and Compression Technique in Wireless Sensor Networks", International Journal of Advanced Research in Computer Science and Software Engineering, Vol. 3, Issue 1, pp.70-73, 2013.

[12] M. Sheik Dawood, L. Ahila, S. Sadasivam, and G. Athisha, "Image Compression in Wireless sensor networks-A survey", International Journal of Applied Information Systems, Vol. 1, No. 9, pp.11-15, February 2012.

[13] Byoungyup Lee1, Myoungho Yeo, Kyungsoo Bok and JaesooYoo, "An Efficient Compression Method in Wireless Sensor Networks", International Journal of Multimedia and Ubiquitous Engineering, Vol. 9, No. 11, pp. 21-30, 2014.

[14] Henry P. Medeiros, Marcos C. Maciel, Richard D. Souza, and Marcelo E. Pellenz, "Lightweight Data Compression in Wireless Sensor Networks Using Huffman Coding", International Journal of Distributed Sensor Networks, Volume 2014, pp.1-11, 2014.

[15] P. RachelinSujae and S. Selvaraju, "Power Efficient Adaptive Compression Techniquefor Wireless Sensor Networks", Middle-East Journal of Scientific Research,Vol. 20, No. 10, pp.1286-1291, 2014.

[16] Asral B. J. and Nor A. Kh., "Performance Comparison of Huffman and LZW Data Compression for Wireless Sensor Node Application", American Journal of Applied Sciences, Vol. 11, No. 1, pp.119-126, 2014.

[17] Maher El Assi1, Alia Ghaddar, Samar Tawbi, GhaddarFadi, "Resource-Efficient Floating-Point Datacompression Using Mas In Wsn", International Journal of Ad hoc, Sensor \& Ubiquitous Computing (IJASUC), Vol.4, No.5, pp.13-28, October 2013.

[18] DragoşIoanSăcăleanu, DragoşMihaiOfrim, RodicaStoian, And VasileLăzărescu, "In node data processing for increasing the lifetime of a wireless sensor network", Proceedings of the 5th WSEAS International Conference on Sensors and Signals (SENSIG '12), pp.7781, September 7-9, 2012, Sliema Malta.

[19] Jonathan GanaKolo, S. AnandanShanmugam, David Wee Gin Lim, Li-MinnAng, and KahPhooiSeng, "An Adaptive Lossless Data Compression Scheme for 
Wireless Sensor Networks", Journal of Sensors, Hindawi Publishing Corporation, Volume 2012, pp.1-20, 2012.

[20] PhayongSornsiriaphilux, DusitThanapatay, KamolKaemarungsi and Kiyomichi Araki, "On Applying Simple Data Compression to Wireless Sensor Networks", International Annual Symposium on Computational Science and Engineering, March 23-26, Chiang Rai, Thailand, 2010.

[21] Andreas Reinhardt, Delphine Christin, Matthias Hollick, Johannes Schmitt, Parag S. Mogre, and Ralf Steinmetz, "Trimming the Tree: Tailoring Adaptive Huffman Coding to Wireless Sensor Networks", Proceedings of the 7th European Conference on Wireless Sensor Networks, No. LNCS 5970, p.33-48, Springer-Verlag, February 2010.

[22] Eug`enePamba Capo-Chichi, Herv`eGuyennet, and JeanMichel Friedt, "K-RLE : A new Data Compression Algorithm forWireless Sensor Network", IEEE Third International Conference on Sensor Technologies and Applications, pp.502-507, 18-23 June 2009, Athens, Glyfada.

[23] P. Sasikala , R.S.D. WahidaBanu, "Extraction of P wave and $\mathrm{T}$ wave in Electrocardiogram using Wavelet
Transform", International Journal of Computer Science and Information Technologies, Vol. 2, No. 1, pp.489493, 2011.

[24] David Huffman, "A Method for the Construction of Minimum Redundancy Codes", Proceedings of the IRE, Vol. 40, Issue 9, pp.1098-1101, 1952.

[25] David Salomon, "Data Compression: The Complete Reference", Springer-Verlag New York, Inc., Third Edition, 2004.

[26] K. Sayood, "Introduction to Data Compression", second edition, Morgan Kaufmann, 2000.

[27] J. J. Rissanen, and G. G. Langdon, "An Introduction to Arithmetic Coding", IBM Journal of Research and Development, Vol. 28, No. 2, pp.135-149, March 1984.

[28] I. H. Witten, J. G. Cleary, and R. Neal, "Arithmetic Coding for Data Compression", Communication ACM, No. 6, pp.520-540, June 1987.

[29] W. R. Heinzelman, A. Chandrakasan, and H. Balakrishnan, "Energy Efficient Communication Protocol for Wireless Microsensor Networks", Proceedings of the 33rd Annually Hawaii International Conference on Systems Sciences, pp.1-10, 2000. 